\title{
Traduire
}

Ine autre perspective sur $r$ tadadciction

Revue française de la traduction

$243 \mid 2020$

Les arts du spectacle

\section{Pour une esthétique de la traduction. Analyse de deux sous-titrages de L'Histoire officielle de Luis Puenzo}

Sylvie Fournié-Chaboche

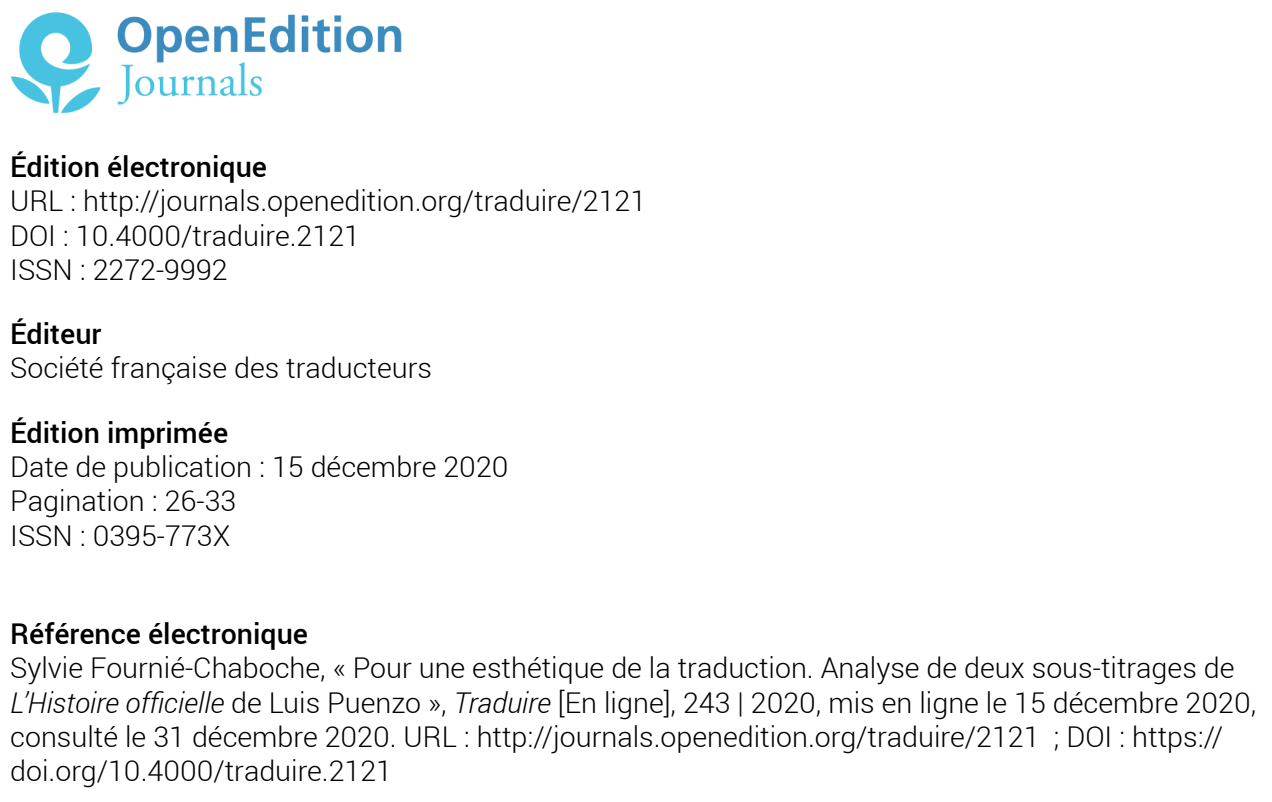




\section{Pour une esthétique de la traduction}

Analyse de deux sous-titrages de L'Histoire officielle de Luis Puenzo

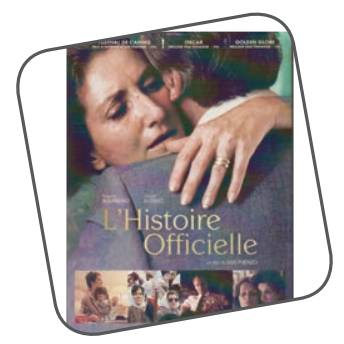

Sylvie Fournié-Chaboche

Alors que les militaires sont encore au pouvoir en Argentine, Luis Puenzo commence à écrire L'Histoire officielle. II en amorce le tournage à l'insu des autorités en 1983, en filmant une manifestation. L'objectif de ce long métrage est résumé par la journaliste Aida Bortnik qui a collaboré à son écriture: «faire un film qui représenterait $95 \%$ du peuple argentin, ceux qui ne savaient pas, qui ne comprenaient pas et qui pensaient n'être ni victimes ni bourreaux» (Lagesse, 2015). Ce film sur le présent des Argentins, sorti en salle en Argentine en avril 1985, est aussitôt présenté et récompensé au Festival de Cannes en mai de la même année.

Cette analyse a pour point de départ la comparaison de deux sous-titrages en français de ce film à trente ans d'écart, l'un réalisé - peut-être faudrait-il dire précipitamment - pour sa présentation au Festival de Cannes, le deuxième accompagnant la version restaurée de 2015. Cela pose d'emblée un certain nombre de questions relatives au rapport entre le sous-titrage et les conditions historiques et 
culturelles de réception de l'œuvre. Le problème est d'autant plus complexe que ce film-témoignage est entretemps devenu un long métrage historique sur un passé dramatique révolu. La nouvelle traduction doit-elle s'adapter à l'inactualité du sujet pour le public étranger trente ans plus tard? Inversement, doit-elle prendre en compte la connaissance a posteriori que ce même public peut avoir des faits qui étaient en train de se tramer?

Cela met notamment en exergue les spécificités du soustitrage cinématographique en tant que technique de traduction appliquée à une œuvre d'art. En effet, la comparaison a surtout révélé des divergences de choix entre traduction et non-traduction de textes fortement symboliques et non dialogués. Au-delà de la compréhension immédiate du message linguistique dans la langue cible, la question du sous-titrage ne doit-elle pas alors être envisagée non seulement du point de vue de la réception, mais aussi de celui de la réceptivité? Cette notion, introduite par Kant dans le domaine de la philosophie, désigne la «faculté de recevoir des représentations, des impressions sensibles, par opposition à la spontanéité de l'entendement» (TLFi). Le sous-titrage appliqué à une œuvre d'art ne doit-il pas lui aussi se mêler d'esthétique?

\section{Un sous-titrage lacunaire, mais étrangement mimétique (1985)}

La version de 1985 comporte un certain nombre d'erreurs manifestes: des problèmes de compréhension de l'espagnol, de cohérence par rapport à la prise de vue, d'ambiguïté, de mise en français. II ne s'agira pas ici de stigmatiser un travail dont l'imperfection trouve certainement une explication dans la précipitation ou le manque de moyens, mais de s'interroger sur le produit fini et d'en analyser l'efficacité.

Il est en effet surprenant que le sous-titrage d'un film, sorti alors que s'ouvre le procès de la junte et qui tente de révéler une amnésie collective, choisisse de ne pas prendre en compte le texte intégral de la chanson de María Elena Walsh «En el país de Nomeacuerdo», chantée à plusieurs reprises par la petite fille, Gaby. Pourtant, cette chanson, censurée par le régime militaire (Borrillo, 2012), est hautement symbolique. Les musiques constituent des éléments de sens très 
importants. La bande originale du film composée par Atilio Stampone, qui revient comme un leitmotiv aux moments les plus poignants, cède la place à deux autres morceaux, "Yesterday» (rebaptisée "Tomorrow»par Ana, revenue d'exil) et cette chanson sur le thème de l'oubli. La portée symbolique de cette dernière est indéniable, surtout quand on découvre que l'enfant est vraisemblablement une fille de disparus, née en captivité et adoptée par une famille proche du pouvoir. On l'entend, en outre, à des moments-clés du film. Quelques minutes après le début, le personnage de Gaby fait son apparition et entonne le premier couplet de cette chanson qui sera terminée par la véritable interprète. Seul le couplet chanté par la fillette est sous-titré: "Au pays du "Ne m'en souviens" / Je fais trois petits pas / Et je me perds / Un petit par-ci / Je ne m'en souviens pas». Plus tard dans le film, la chanson originale fredonnée tient lieu de fond musical, quand Alicia, la mère de Gaby, partant à la recherche de l'histoire de sa fille adoptive, tente de trouver des indices sur les langes que l'enfant portait quand on la lui a amenée. La chanson déborde alors sur la scène suivante qui se déroule dans la maternité où Alicia mène son enquête et se mêle aux cris des femmes qui accouchent: l'oubli volontaire de la naissance de Gaby (vraisemblablement en captivité avant la disparition de ses parents) est donc rappelé ici par cette superposition de sons, les cris venant progressivement remplacer la douceur du refrain. La chanson est à nouveau entendue au téléphone lors d'un appel de la fillette à ses parents, à la toute fin du long métrage. Seul le début des paroles "Au pays du "Ne m'en souviens" "est sous-titré. L'Histoire officielle s'achève sur un plan de Gaby s'endormant en reprenant la mélodie (non sous-titrée). Celle-ci se trouve ensuite fredonnée en fond musical au générique de fin.

S'il reste surprenant qu'un élément aussi important du film que cette chanson n'ait pas été sous-titré entièrement, force est de constater que cette même économie de sous-titres touche d'autres textes qui ont tous en commun d'être non dialogués. C'est notamment le cas pour les clameurs des célèbres manifestations de la place de Mai, tournées clandestinement en prise de vue réelle. Le film les évoque dans deux scènes entremêlant chacune chants, slogans et textes écrits sur des banderoles ou pancartes. La première fois, tous 
ces éléments sont traduits dans les deux versions. Pour la deuxième manifestation, la version de 1985 ne présente pratiquement aucun sous-titre. Seuls deux slogans s'inscrivent sur l'écran: "Ni oubli ni amnistie / rendez-les vivants» et "ll est indispensable qu'on nous les rende vivants et qu'on châtie les coupables». Curieusement, ces slogans sont criés bien avant qu'ils n'apparaissent sous-titrés à l'écran. Ils se trouvent donc associés à une image qui n'a rien à voir, montrant des Argentins qui brandissent une banderole sur laquelle on devine le texte suivant: Familiares de desaparecidos y detenidos por razones politicas [Familles de disparus et de prisonniers politiques]. Un spectateur ignorant de l'espagnol serait légitime à penser qu'il s'agit de la traduction de la banderole.

Les pancartes et banderoles de cette deuxième manifestation ne sont pas traduites. Pas même celle brandie par une grand-mère de la place de Mai, Sara (peut-être la grandmère de Gaby), montrant la photo de sa fille enceinte et de son gendre. Sur le ventre de sa fille disparve est écrit y su bebe nacido en cautiverio [et son bébé né en captivité].

Dans le premier sous-titrage, on constate donc une tendance à ne pas appliquer le même traitement aux textes dialogués dans lesquels se développe l'intrigue et à ceux, non dialogués, souvent non fictifs (documents, prises de vue réelles, chanson existante). Ces derniers semblent avoir été traités comme des objets folkloriques qui intéressent le traducteur plus par leur présence, dont il faut rendre compte au moins une fois, que par leur contenu (au point de condenser tous les slogans en deux phrases placées de façon presque aléatoire dans l'épisode de la manifestation).

À l'instar de l'histoire officielle crue et enseignée par l'héroïne Alicia, professeur d'histoire, en ne s'appuyant que sur les écrits autorisés et en ignorant toutes les sources d'informations extérieures (manifestations, presse, chahut de ses élèves), le sous-titrage d'origine (1985), dans un curieux mouvement mimétique, semble donc ne prendre en compte de façon privilégiée qu'une seule forme "d'histoire» (diégèse): celle qui a été écrite par les auteurs et transformée en dialogues. Ce qui appartient au réel (la chanson de María Elena Walsh et les scènes tournées sur le vif) est plus facilement passé sous silence. 


\section{Un sous-titrage complété et orienté vers la réception du fait historique (2015)}

Luis Puenzo a confié ne pas avoir immédiatement mesuré la portée de son film qui connut d'abord le succès au Festival de Cannes, alors qu'il était passé inaperçu en Argentine à sa sortie. En mars 1986, il obtient la consécration, en remportant l'Oscar du meilleur film étranger. L'œuvre fait alors office de catharsis dans le paysage cinématographique argentin muselé par des années de dictature. Bien sûr, l'émotion suscitée ne peut être la même en 1985 en Argentine et à Cannes, et encore moins trente ans plus tard en France. On imagine bien la déflagration qu'a pu représenter ce film qui donnait à voir l'aveuglement collectif à travers le personnage d'Alicia, professeur enseignant l'histoire officielle et ouvrant peu à peu les yeux sur la réalité de son pays.

Dans la version de 2015, le parti pris de traduction initial est totalement inversé. Qu'il s'agisse de la chanson, des slogans, des pancartes ou des banderoles, on passe en effet d'une traduction a minima à un sous-titrage intégral, tandis que dans la première version, les scènes de la vie réelle semblaient se suffire à elles-mêmes. En raison de la distanciation temporelle ou spatiale du public par rapport au tournage, on a tendance à croire que plus il y aura d'informations tangibles (donc sous-titrées), meilleure sera la réception du film. Peut-être craint-on de ne pas tout donner à percevoir, car le spectateur actuel est vu en historien avide de données et sachant tirer les leçons du passé ou, au contraire, est jugé trop ignorant du sujet pour pouvoir l'apprécier.

Ce phénomène est particulièrement flagrant à la fin du film, avec la chanson "En el país de Nomeacuerdo» qui, cette fois, est entièrement sous-titrée. La traduction diffère d'ailleurs légèrement par rapport à celle de 1985: "Au pays du je-ne-me-souviens-plus». Elle est alors chantée par Gaby au téléphone et constitue l'arrière-plan musical de la scène qui se déroule sous les yeux du spectateur, elle-même relativement sonore (Alicia rassemble ses affaires pour quitter la maison). On s'emploie là véritablement à faire en sorte que la portée symbolique de la chanson n'échappe pas au spectateur. C'est un progrès incontestable pour la réception du film par le public. Cependant, on observe une disproportion 
entre ces caractères en surbrillance qui viennent illuminer une scène se déroulant dans l'obscurité et le filet de voix à peine audible de Gaby au bout du fil. Du fait du sous-titrage qui empiète sur l'image, l'équilibre entre le son et l'image se trouve perturbé, tout comme le plaisir du spectateur d'avoir réussi à créer des liens, à saisir les éléments qui se font écho, contribuant à l'esthétique de l'œuvre.

\section{Pour une esthétique de la traduction orientée vers la réceptivité}

Cette analyse nous amène donc à nous interroger sur les spécificités de la traduction appliquée à l'œuvre d'art. Celle-ci est bien sûr indissociable de sa réception et de son contexte. Les omissions de la traduction de 1985 (comblées dans la version de 2015) pourraient, certes, trente ans après, sembler incongrues au spectateur français qui regarderait ce film avec la curiosité d'un historien en quête d'informations et de réseaux de symboles compréhensibles a posteriori. Faut-il pour autant condamner cette version originale?

Est-il toujours légitime de plaquer un écrit venu d'ailleurs sur certaines scènes qui font sens bien au-delà du sens littéral? Prenons simplement l'exemple de la banderole sur laquelle on arrive à lire Familiares de desaparecidos y detenidos por razones politicas; est-il véritablement utile d'insérer en surbrillance "les familles des disparus et des prisonniers politiques» comme le fait la version restaurée? Outre la proximité des langues latines qui permet un décryptage plus ou moins précis du texte, ne doit-on pas laisser s'exprimer l'œuvre d'art de façon autonome lorsqu'elle peut le faire? N'est-il pas plus efficace pour le spectateur d'essayer de déchiffrer cette banderole au gré de ses déplacements, comme le fait Alicia qui tente de découvrir la réalité dans laquelle elle vit? La solution facile et immédiate du soustitrage est d'une certaine façon incompatible avec le jeu que toute œuvre d'art entretient avec son spectateur. L'œuvre d'art ne vise pas la compréhension rapide et sans ambiguïté. Elle veut produire une émotion esthétique, même si celle-ci est difficile d'accès. Les prises de vue réelles ont déjà tellement de valeurs connotatives (Genette, 1987) qu'elles n'ont pas forcément besoin systématiquement de mots pour les 
décrire. N'oublions pas que des textes filmés restent avant tout des images...

C'est donc bien aussi du point de vue de la réceptivité (création d'impressions), et non de la seule réception, qu'il faut envisager les tentatives de traduction d'une œuvre d'art.

\section{Conclusion}

L'analyse des sous-titrages du film La Historia Oficial de Luis Puenzo invite à réfléchir à la traduction cinématographique en tant qu'œuvre d'art.

Le sous-titrage d'origine semble relativement incomplet. Toutefois, l'on peut se demander si, dans la version restaurée, la traduction systématique de tout texte écrit et oral (dialogué, chanté, crié) n'a pas tendance à étouffer le film, à affadir des images qui parlent d'elles-mêmes, ou encore à créer un déséquilibre dans la combinaison image-son, comme dans la scène où une chanson fredonnée au loin devient un élément visuel éclatant sur une prise de vue sombre.

Si l'on admet que la traduction d'une œuvre d'art doit tenir compte autant de la réceptivité que de la réception, alors le traducteur doit se mêler d'esthétique. II doit choisir de traduire ou non en fonction de critères relevant de la beauté, de l'émotion, et non pas seulement de la compréhension littérale.

Nous avons constaté un curieux parallélisme entre la nature des textes non traduits (souvent des scènes réelles) et l'aveuglement d'Alicia au début du film. Quelles que soient les raisons qui ont présidé à l'absence de traductions, les «silences de la traduction» associés aux prises de vue réelles ne sontils pas aussi l'expression la plus aboutie de l'impossibilité pour les Argentins de s'interroger sur ce que la raison ne pouvait admettre (enlèvements d'enfants, tortures)? Ces vides ne traduisent-ils pas justement auprès du spectateur français de l'époque, et bien mieux que des mots, le malaise que l'on ressent à voir sans comprendre, surprenante mise en abyme du film?

sylvie.fournie@univ-orleans.fr 
Sylvie Fournié-Chaboche est enseignante-chercheuse en espagnol à l'université d'Orléans. Membre du Laboratoire ligérien de linguistique (UMR7270), elle consacre ses recherches à la lexicographie, la phraséologie, la traduction des expressions idiomatiques et à leur emploi dans le discours littéraire, notamment dans l'œuvre de Javier Tomeo dont elle est spécialiste. Elle est responsable de l'ensemble des enseignements de traduction espagnol/français au sein de la spécialité «Traduction et communication multilingue» du master «Langues et Sociétés».

\section{SOURCES CITÉES}

BORRILlO Daniel, Interlude, La Revue des Droits de l'Homme, n 2, 2012, https://doi. org/10.4000/revdh.254, consulté le 4/11/2020.

GENETTE Gérard, Seuils, Paris, Seuil, coll. «Poétique», 1987.

KANT Emmanuel, Critique de la raison pure, Paris, Gallimard, 1980.

LAGESSE Éric (dir.), «L'Histoire officielle», livret accompagnant la version restaurée, Édition DVD Pyramide Vidéo, 2015

PUENZO Luis, «L'Histoire officielle», L’Avant-Scène Cinéma, n 350, 1986.

SÁNCHEZ GELÓS Verónica (dir.), La Historia Oficial, Instituto Nacional de Cine y Artes Audiovisuales (INCAA), Buenos Aires, 2015. Entretiens recueillis par Patricia Moro.

Trésor de la Langue française informatisé (TLFi), http://atilf.atilf.fr/ 